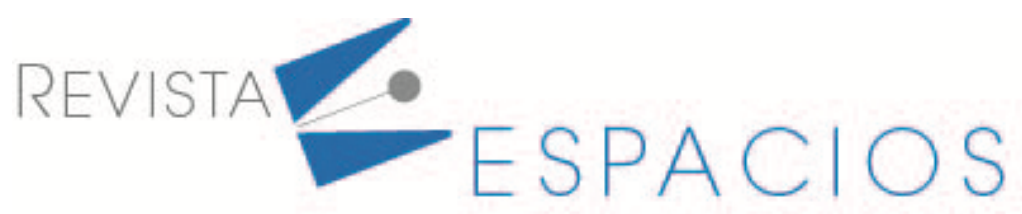

\title{
Identificación de herramientas tecnológicas usadas por la comunidad Wayuu en La Guajira (Norte de Colombia)
}

\author{
Identification of technological tools used by the Wayuu population in La Guajira (Northern \\ Colombia)
}

\author{
MUÑOZ, Delvis ${ }^{1}$ \\ MARTELO, Raul J.2 \\ CURIEL, Rebeca Y. ${ }^{3}$
}

\begin{abstract}
Resumen
Se identificaron las herramientas tecnológicas usadas por la población Wayuu con el fin de superar las barreras y dificultades presentadas para su manejo en el corregimiento de Nazareth Municipio de Uribía, Departamento de La Guajira, Colombia. La técnica utilizada para la recolección de información fue la encuesta. Se diseñó como instrumento un cuestionario auto administrado, conformado por preguntas cerradas, enmarcadas en cinco alternativas de respuesta tipo escala de Likert. Se concluye que existe baja presencia en herramientas tecnológicas, estas pueden ser consideradas para algunos como un peligro a la identidad, significando pérdida de las tradiciones ancestrales, poco conocimiento para manejar las herramientas TIC.

Palabras clave: tics, comunidad indígena, herramirntas tecnológicas, wayuu
\end{abstract}

\begin{abstract}
The technological tools used by the Wayuu population of the township of Nazareth, Municipality of Uribía, Department of La Guajira, Colombia, were identified. The technique used to collect information was the survey. A self-administered questionnaire was designed as an instrument, made up of closed questions, framed in five Likert scale response alternatives. It is concluded that there is a low presence in technological tools, these may be considered by some as a danger to identity, meaning loss of ancestral traditions, coupled with the risk of unsustainability.

Key words: icts, indigenous community, technological tools, wayuu
\end{abstract}

\section{Introducción}

Las herramientas tecnológicas han transformado totalmente la forma en la que nos comunicamos con los demás, proporcionándonos herramientas para conversar, compartir imágenes, archivos, entre otros (Neme y Arias, 2019). No hay duda de que nos han facilitado la vida y conocimiento dentro y fuera de las organizaciones. Componente importante es la tecnología, ya que en ella podemos ver el resultado de la actividad que imparte el hombre en sociedad en procura de la satisfacción de sus necesidades y deseos (Rodríguez et al., 2017). Las herramientas tecnológicas son dispositivos o programas diseñados que facilitan el trabajo, además permiten la

\footnotetext{
${ }^{1}$ Docente de Planta Tiempo Completo. Universidad de La Guajira. Riohacha, La Guajira, Colombia. Email: dmunozr@uniguajira.edu.co

${ }^{2}$ Docente de Planta Tiempo Completo. Universidad de Cartagena. Cartagena de indias, Bolivar, Colombia. Email: rmartelog1@unicartagena.edu.co

${ }^{3}$ Docente de Planta Tiempo Completo. Universidad de La Guajira. Riohacha, La Guajira, Colombia. Email: rcuriel@uniguajira.edu.co
} 
aplicación de los recursos de manera efectiva, ya sea intercambiando información y conocimiento dentro o fuera de las organizaciones (Ochoa et al., 2020; Chisag et al., 2017). En el mismo sentido, las herramientas tecnológicas han tomado mayor importancia, ya que han permitido superar diversas barreras, y ahorrar tiempo al momento de completar las actividades, además de que su uso ha sido clave para la obtención de resultados óptimos y disminución de costos (Acevedo \& Flórez, 2019).

Una de las principales herramientas tecnológicas con alta frecuencia de uso son las redes sociales. Una red social es un sitio en internet que tiene como finalidad servir herramientas de comunicación entre diversos usuarios que se unen en un mismo espacio virtual para compartir información en diversos formatos como textos, videos, imágenes, música, entablando diálogos sobre algún interés o tema en común(Jiménez et al., 2019). Asimismo, las redes sociales y los espacios virtuales son mecanismos de interacción, simples canales de comunicación, pero con un potencial de alcance y de impacto mucho mayor que cualquiera de los otros medios tradicionales, y con capacidad de interacción y dinamismo, superiores a cualquiera de los otros medios (Martínez et al., 2013).

Por otra parte, Internet es una herramienta que avanza a una gran velocidad. A veces no conseguimos captar de manera inmediata los cambios que sufre y las oportunidades que ofrece, lo que nos genera inseguridad y desconfianza. Sin embargo, cuando nos disponemos a disfrutar, aprender, crear, compartir, facilitar, impulsar, podemos hablar de espacio de confianza (Núñez, 2017). Además, Internet ha permitido poner al alcance de jóvenes y mayores una gran cantidad de temas culturales provienen de cualquier parte del mundo son virtuales y, en ocasiones, con fines delucro. Para que un producto cultural, llámese libro, película, espectáculo, videojuego o anuncio, ayude a volver sociable a un joven o a humanizar a un adulto para ser adaptado a ellos. Cuando no hay adaptación, los aportes convierten a los consumidores en sujetos pasivos (Manfredi, 2020). Los problemas culturales sugieren llevar a cabo acciones para aprovechar las oportunidades que la globalización ofrece (Kischinhevsky, 2020).

De igual manera, los Entornos Virtuales de Aprendizajes (EVA), son el conjunto de medios de interacción sincrónica y asincrónica que, con base en un programa curricular, lleva a cabo el proceso de enseñanza y aprendizaje a través de un sistema de administración de aprendizaje (Perez \& Aguilar, 2020). El uso de las Tecnologías de la Información y la Comunicación (TIC) en la educación, ha implicado una serie de cambios significativos en el proceso de Enseñanza y Aprendizaje (Zelada, 2020). Dentro de estos cambios significativos puede resaltarse la creación de Entornos Virtuales de Aprendizaje, los cuales ofrecen la posibilidad de romper las barreras de espacio y tiempo que existen en la educación tradicional y posibilitan una interacción abierta a las dinámicas del mundo educativo (Castañeda \& Selwyn, 2020).

Por su parte, Becerra \& Gómez, (2020) afirman que, los centros TIC son lugares donde los ciudadanos procesan los miedos de acercarse a la tecnología, en ellos también se caen mitos individuales y se potencializan expectativas sobre el manejo de herramientas tecnológicas. Igualmente, son espacios de encuentros para el intercambio de conocimiento y la construcción de apuestas colaborativas encaminadas a mejorar las condiciones de vida del entorno; y contribuir al reconocimiento y a la inclusión social de poblaciones como los jóvenes, las mujeres, las personas mayores, las personas con discapacidad, entre otras. Sin duda, la creación de entornos virtuales de aprendizaje nos ha dado la ventaja de fragmentar las barreras y la xenofobia sobre el desconocimiento por las nuevas tecnologías que existen en las aulas tradicionales y facilitan una interacción abierta a las dinámicas del mundo y los retos de formación resultan claves en entornos donde el conocimiento, así como las oportunidades y los servicios sociales, están distribuidos de manera desigual.

Finalmente, los indígenas Wayuu poseen particularidades entre los grupos étnicos de Colombia, en primer lugar, contrario a los grupos indígenas que han atravesado un proceso de recuperación, reconstrucción o renacer indígenas, estos individuos poseen una fuerte trayectoria de conciencia étnica, es decir, gran parte de sus individuos siempre se han identificado como Wayuu. En segundo lugar, conforman un grupo étnico que pese a 
adoptar muchos elementos de culturas extranjeras, han gozado de cierta independencia gracias a su posición geográfica y a su resistencia frente a los órdenes impuestos por los civilizados. Tercero, son un grupo muy numeroso, que pese a estar hoy asediados por el narcotráfico, no son un grupo en vía de extinción (como es el caso de varios grupos indígenas de Colombia). En cuarto lugar, los Wayuu son un una comunidad socialmente heterogénea, no existe entre ellos un solo modelo o líder Wayuu que represente a todas las personas que se denominancomo tales, lo que sugiere que no existe un solo modo de vida Wayuu, por que ellos cuentas con sus propias culturas ancentrales y darle paso a las TIC sienten que pueden ser aculturizados por abrir espacios a medios tecnológicos que no pertenecen a sus costumbres (Dlestikova, 2020).

Con base a lo anterior se explica, la sociedad Wayuu de hoy es muy compleja y debe abrirse el compás de análisis e ir más allá de la simple observación de la cotidianidad de una ranchería o caserío. También en buscar las dinámicas que establecen intercambios culturales y además ver de qué manera entran en el juego de la estructura de la nación y de sus diferentes instituciones. Debido a todo lo expuesto en este trabajo se buscó Identificar las herramientas tecnológicas usadas por la población Wayuu del corregimiento de Nazareth Municipio de Uribía Departamento de La Guajira, Norte de Colombia.

\section{Metodología}

El trabajo es de tipo descriptivo con diseños de investigación de campo no experimental, además como se realiza en un momento preciso y en un tiempo determinado se considera transaccional o transversal, ya que el investigador estudia el evento en un único momento del tiempo.

\subsection{Población}

Los sujetos claves estuvieron conformados por la población Wayuu del corregimiento de Nazareth Municipio de Uribía Departamento de La Guajira (Colombia), a quienes se le aplicaron los instrumentos de recolección de datos: La población de estudio fueron 90 habitantes de la población Wayuu del corregimiento de Nazareth. En la Tabla 1, se distribución la población Wayuu de la siguiente manera:

Tabla 1

Distribución de la población

\begin{tabular}{ccc}
\hline Población Wayuu & Grupo de edades & Encuestas/grupo por edades \\
\hline Adolescentes & $10-17$ & 30 \\
\hline Adulto Joven & $18-44$ & 40 \\
\hline Adulto Mayor & $45-75$ & 20 \\
\hline Total, Encuestas/grupo por edades & 90 \\
\hline
\end{tabular}

Fuente: Elaboración propia

\subsection{Técnicas e instrumentos para la recolección de datos}

La técnica utilizada para la recolección de información fue la encuesta tipo Likert; seleccionando el cuestionario como instrumento para ser aplicado en la población objeto del estudio (Vargas et al., 2019). Se diseñó como instrumento un cuestionario auto administrado; a la población wayuu del corregimiento de Nazareth. El instrumento está conformado preguntas cerradas, enmarcadas en cinco (5) alternativas de respuesta clasificadas como: Totalmente de Acuerdo (5), De acuerdo (4), Ni de acuerdo ni en desacuerdo (3), En desacuerdo (2) y Totalmente en Desacuerdo (1). En este sentido, se construyó una tabla de puntaje, rango, niveles y categoría para el análisis del promedio, sobre la base de alternativas de respuestas que fueron cinco (5) y en consideración a estas alternativas planteadas se elaboró un baremo para la interpretación de las variables de estudio. 


\subsection{Análisis de los datos}

El análisis de los datos obtenidos a través del cuestionario se realizó con el software IBM SPSS statistics 22 . Al tratarse de variables categóricas se utilizó el procedimiento frecuencias gráficas y además medidas de tendencia central: Media, mediana y moda, según Bedoya et al., (2019) las técnicas de análisis de datos usadas. Los datos recolectados mediante la aplicación de los instrumentos fueron procesados con estadísticas descriptivas.

Por otra parte, se construyó el baremo de análisis, con el fin de interpretar el cuestionario y determinar la calificación de las variables objetos de estudio. En efecto a continuación la tabla 2 contiene la codificación elaborada para establecer los puntajes o calificaciones, así como las categorías de análisis elegidas que sirvieron para ubicar el comportamiento de la variable estudiada a partir del puntaje acumulado por los indicadores y las dimensiones. A continuación, se muestra el baremo para la interpretación de la media en los resultados de la investigación el cual se construyó seleccionándose cuatro categorías de análisis con sus correspondientes intervalos de interpretación.

$$
\text { Valor Mayor - Valor Menor 5-1 = 4/4 = } 1
$$

Para construir los intervalos, se empieza con el mayor valor asignado a las alternativas de respuesta y se resta el menor valor asignado a estas dividiéndose luego entre el número de categorías a seleccionar. Esta operación permite construir el rango de interpretación, condición necesaria para mostrar el nivel de presencia del indicador y dimensión analizado, se establecieron dos baremos de ponderación, Tabla 2 y 3, para luego ser utilizados en el análisis de las dos variables.

Tabla 2

Baremo para la interpretación de la media

\begin{tabular}{cc}
\hline Rango & Categoria de analisis \\
\hline $5-4,2$ & Alta \\
\hline $4,21-3.41$ & Moderadamente Alta \\
\hline $3,42-2.62$ & Moderada \\
\hline $2,63-1,83$ & Moderadamente Baja \\
\hline $1.8-1$ & Baja \\
\hline
\end{tabular}

Fuente: Ruiz et al. (2020)

En lo que respecta a la medida de variabilidad, se utilizó la desviación estándar, esta última para indicar el grado de dispersión de la respuesta con relación a la escala de medición utilizada y su rango, representada por las puntuaciones mayor y menor obtenidos, es decir, cinco (5) y uno (1), la cual permitió elaborar para su respectivo análisis la siguiente Tabla 3 de rango, intervalo y categoría.

Tabla 3

Baremo para la interpretación de la desviación estándar

\begin{tabular}{c|c|c}
\hline & Intervalo & Categoria \\
\hline 5 & $1,09-1,21$ & Alta dispersion \\
\hline 4 & $0,97-1,08$ & Moderadamente Alta dispersion \\
\hline 3 & $0,85-0,96$ & Moderada dispersion \\
\hline 2 & $0,72-0,84$ & Moderadamente Baja dispersion \\
\hline 1 & $0,60-0,71$ & Baja dispersion \\
\hline
\end{tabular}

Fuente: Elaboración propia 


\section{Resultados}

Se procedió a calcular la media aritmética y la desviación estándar, así como las categorías correspondientes de los indicadores que integraron esta dimensión identificados como: redes sociales, Internet, y entornos virtuales de aprendizajes, obteniendo los resultados representados a continuación en la Tabla 4.

Tabla 4

Herramientas Tecnológicas

\begin{tabular}{|c|c|c|c|c|c|c|c|c|c|c|c|c|c|}
\hline \multirow{2}{*}{\begin{tabular}{|l} 
Cate goria de \\
la Res spues ta \\
Indicador \\
\end{tabular}} & \multicolumn{2}{|c|}{1} & \multicolumn{2}{|c|}{2} & \multicolumn{2}{|c|}{3} & \multicolumn{2}{|c|}{4} & \multicolumn{2}{|c|}{5} & \multirow{2}{*}{ 裉 } & \multirow{2}{*}{ 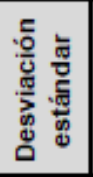 } & \multirow{2}{*}{ 뜽 } \\
\hline & $\mathrm{Fa}$ & $\%$ & $\mathrm{Fa}$ & $\%$ & $\mathrm{Fa}$ & $\%$ & $\mathrm{Fa}$ & $\%$ & $\mathrm{Fa}$ & $\%$ & & & \\
\hline \begin{tabular}{|l} 
Redes \\
Sociales
\end{tabular} & 6 & 6,7 & 48 & 53,3 & 18 & 20 & 16 & 17,8 & 2 & 2,2 & 2,56 & 0,937 & \begin{tabular}{|l} 
Media \\
Dispersion
\end{tabular} \\
\hline Internet & 6 & 6,7 & 45 & 50 & 18 & 20 & 14 & 15,6 & 7 & 7,8 & 2,69 & 1,098 & \begin{tabular}{|l|} 
Muy Alta \\
Dispersion
\end{tabular} \\
\hline \begin{tabular}{|l} 
Entorno \\
virtuales de \\
Aprendizaje
\end{tabular} & 8 & 8,9 & 53 & 58,9 & 20 & 22,2 & 7 & 7,8 & 2 & 2,2 & 2,36 & 0,839 & \begin{tabular}{|l} 
Baja \\
Dispersion
\end{tabular} \\
\hline Total & 7 & 7 & 49 & 54 & 19 & 21 & 12 & 14 & 4 & 4 & & & \\
\hline \multicolumn{4}{|c|}{ Promedio de la Dimensión } & \multicolumn{7}{|c|}{2,54} & & & \\
\hline \multicolumn{4}{|c|}{ Categoria de la Dimensión } & \multicolumn{7}{|c|}{ Baja Presencia } & & & \\
\hline \multicolumn{4}{|c|}{ Promedio desviación estándar } & \multicolumn{7}{|c|}{0,958} & & & \\
\hline \multicolumn{4}{|c|}{ Categońa desviación estándar } & \multicolumn{7}{|c|}{ Media Dispersion } & & & \\
\hline
\end{tabular}

Fuente: Elaboración propia

Los resultados obtenidos en la Tabla 4, demostraron que el indicador con mayor presencia en las herramientas tecnológicas fue el internet, cuya media es 2,69; seguido en orden de presencia por la herramienta redes sociales con una media de 2,56; a esta le sigue Entornos Virtuales de Aprendizajes, Con media de 2,36; el indicador internet según baremo para la media, estuvo en la categoría de mediana presencia, mientras que redes sociales y Entornos Virtuales De Aprendizajes estuvieron en baja presencia.

A su vez, los valores obtenidos para la desviación estándar fueron: 0.937, 1.098 y 0.839 respectivamente, considerados según baremo de la desviación estándar, para la primera y tercera herramientas, media dispersión y baja dispersión, por tanto, intermedia e baja confiabilidad de los resultados y para la segunda herramienta las dispersiones se catalogaron como muy alta dispersión considerando muy alta la confiabilidad de las respuestas.

De esa manera, la dimensión herramientas tecnológicas, obtuvo un valor promedio de 2,54, correspondiéndole una categoría de bajo nivel de presencia en la población estudiada, con una desviación estándar de 0.958, indicando una mediana dispersión de los datos, considerado este resultado de confiabilidad media. Representando de esta manera, la primera dimensión en cuanto al nivel de presencia dentro de la variable estudiada, la cual demanda ser medianamente fortalecida en la población Wayuu del corregimiento de Nazareth.

Por su parte, la mediana presencia del indicador internet coincide con Mancinas \& Nogales, (2014), quienes explican que internet es una herramienta que avanza a una gran velocidad. A veces no conseguimos captar de manera inmediata los cambios que sufre y las oportunidades que ofrece, lo que nos genera inseguridad y desconfianza. Sin embargo, cuando nos disponemos a disfrutar, aprender, crear, compartir, facilitar, impulsar, podemos hablar de espacio de confianza.

Sin embargo, el bajo nivel de presencia de los demás indicadores, redes sociales, entornos virtuales de aprendizajes, contrastan con los postulados de Villalobos (2016) que afirma una red social es un sitio en internet que tiene como finalidad el servir herramientas de comunicación entre diversos usuarios que se unen en un 
mismo espacio virtual para compartir información en diversos formatos como textos, videos, imágenes, música. Mendoza y Martínez (2020) manifiestan que el uso de las Tecnologías de la Información y la Comunicación en la educación, ha implicado una serie de cambios significativos en el proceso de Enseñanza y Aprendizaje.

Por tal razón, la baja presencia de la dimensión herramienta tecnológica se debe a:

- La mayoría de la población tiene poco conocimiento del uso de estas herramientas, además,

- Por su cultura y forma de enseñanza no está presente utilizar la tecnología.

- Desconocen no recibir una formación educativa u capacitaciones que oriente al Wayuu para la utilización de las TIC que les permita crear, guardar, procesar y usar la información para luego compartirla. De igual manera, las limitaciones sobre su uso dificultan la obtención de estas tecnologías

- Los bajos recursos que poseen. Tampoco, existe un espacio adecuado en donde se propicie las practica al uso de cualquier tipo de dispositivos en referencia a la tecnología en el momento que se requiera, igualmente

- Existen dificultades en cuanto a los servicios públicos en especial con la energía eléctrica que es fundamental para la implementación de este proyecto, por lo que, corresponde a una deficiencia para ejecutarlo, de acuerdo a lo dicho por Torres et al (2020) en el sentido de que las herramientas tecnológicas han tomado mayor importancia, ya que nos han permitido superar diversas barreras, y ahorrar tiempo al momento de completar las actividades.

\section{Conclusiones}

Analizados los resultados y su discusión, se presenta con baja presencia las herramientas tecnologicas, donde se expone que el Wayuu mantienen su identidad, en especial el idioma y sus ancestrales, además, manifiestan, no recibir educación sobre las TIC, mas cuando existen dificutades para colocar estos dispositivos, cuando tampoco se tiene conocimiento para manipularlos y las dificultades que tienen con los servicios publicos en especial con la energia electrica.

Por lo tanto, toda esta situación que se presenta con referencia a las herramientas tecnológicas y a las razones presentadas, se hace necesario que sea intervenida por el Estado, para que la población Wayuu del corregimiento de Nazareth pueda mejorar su situación, y de esta manera, adecuar los lugares propicios para implementar estas herramientas con el fin de que la población pueda ser educada por estos medios y recibir, otra clase de servicios que son fundamentales para el desarrollo de su vida cotidiana.

El identificar las herramientas usadas por la población Wayuu entre las que se encuentran el internet, es de importancia, debido a que su conocimiento le permite superar las barreras y dificultades presentadas para su manejo contribuyendo a reorientarlo para que su uso sea más efectivo en sus diferentes labores.

Asimismo, para los lideres Wayuu y personal administrativo del corregimiento de Nazareth y del Municipio de Uribia, señalan que es la puerta al éxito, la posibilidad de vencer las brechas digitales, en minimizar los rezagos económicos y las exclusiones de la población Wayuu del corregimiento de Nazareth.

\section{Referencias bibliográficas}

Acevedo-Rincón, J. P., \& Flórez-Pabón, C. E. (2019, January). TelEduc: A virtual learning environment for teaching and learning at the University of Campinas, Brazil. In Journal of Physics: Conference Series (Vol. 1161, No. 1, p. 012023). IOP Publishing. 
Becerra Patiño, L. M., \& Gómez Hernández, J. F. (2020). Explorando otros mundos: entre lo vivido y lo enseñado en la Escuela Rural de Galdámez.

Bedoya, Elías A., Vargas-Ortiz, Luz E., Severiche-Sierra, Carlos A., \& Meza-Aleman, María de J.. (2019). Modelo Logit para la Presencia de Problemas Osteomusculares en Trabajadores del Sector Hospitalario. Información tecnológica, 30(2), 181-188.

Castañeda, L., \& Selwyn, N. (2020). Reiniciando la universidad: buscando un modelo de universidad en tiempos digitales. Editorial UOC.

Chisag, J. C. C., Lagla, G. A. F., Alvarez, G. S. V., Moreano, J. A. C., Pico, O. A. G., \& Chicaiza, E. M. I. (2017). Utilización de recursos didácticos interactivos a través de las TIC' $S$ en el proceso de enseñanza aprendizaje en el área de matemática. Boletín Redipe, 6(4), 112-134.

Dlestikova, T. (2020). Encuentros entre las Justicia Indígena y Restaurativa en Colombia. Novum Jus, 14(1), 1540.

Jiménez, M. Á. S., de Matos, N., \& Correia, M. B. (2019). Evolution of the Presence and Engagement of Official Social Networks in Promoting Tourism in Spain. Journal of Spatial and Organizational Dynamics, 7(3), 210225.

Kischinhevsky, M. (2020). De las síntesis informativas a los resúmenes para altavoces inteligentes, desafíos al periodismo radiofónico de carácter local1. Estudios sobre el mensaje periodístico, (26), 167-175.

Mancinas-Chávez, R., \& Nogales Bocio, A. I. (2014). Primer Congreso Internacional Infoxicación: mercado de la información y psique, Libro de Actas. Sevilla: Ladecom, Facultad de Comunicación, Universidad de Sevilla.

Manfredi-Sánchez, J. L. (2020). Globalización y poder: la consolidación de la comunicación internacional como disciplina. Artículo de revisión. El profesional de la información, 29(1).

Martínez, R., Corzana, F., \& Millán, J. (2013). Experimentando con las redes sociales en la enseñanza universitaria en ciencias. Revista Eureka sobre enseñanza y divulgación de las ciencias, 10(3), 394-405.

Mendoza, L. R. M., \& Martínez, M. E. M. (2020). TIC y neuroeducación como recurso de innovación en el proceso de enseñanza y aprendizaje. ReHuSo: Revista de Ciencias Humanísticas y Sociales, 5(2), 85-96.

Neme, A., \& Arias, L. (2019). Influencia de las redes sociales en la construcción de la identidad de los estudiantes universitarios. Entropia, 3(5), 195-214.

Núñez, Q. Á. (2017). Pedagogía sistémica e interculturalidad: claves para construir un aula inclusiva. Revista Lusófona de Educação, (37), 165-179.

Ochoa, A. L. G., Ramírez, J. G. M., Hernández, M. E. T., \& Rizo, A. S. (2020). Influencia de las TIC en el proceso administrativo. Revista Científica de FAREM-Estelí, (33), 52-63.

Pérez, M. M. D., \& Aguilar, B. L. C. (2020). Estudio para realizar la acción tutorial a través de un sistema de gestión de aprendizaje en Moodle para el nivel de secundaria. MLS Educational Research, 4(1).

Rodríguez, C. A., Calatayud, M. M., \& Valero, T. N. (2017). The Martell Rumbaut family's social networks in the fishing community of Castillo-Perché, Cuba. Maguaré, 31(1), 87.

Ruiz, M., Severiche, C., Briceño, L., Duran, L. (2020). Barreras de Competitividad de las PyMEs Agrícolas del Distrito de Santa Marta (Caribe Colombiano). Revista Espacios, 41(17), Pág. 6. 
Torres, O. H., Tamayo, P. Á. L., \& Ovalle, C. Á. R. (2020). La mediación pedagógica de las Tecnologías de la Información y la Comunicación: una vía para el desarrollo cultural del escolar rural.(Revisión). Roca. Revista científico-educacional de la provincia Granma, 16, 438-450.

Vargas-Ortiz, L. E., Villalba-Vimos, V. V., Severiche-Sierra, C., \& Padilla, H. E. (2019). TICs y gestión de la innovación en MiPyMEs: Un análisis con experimentos factoriales para las utilidades. Revista Espacios, 40 (13), Pág. 24

Villalobos, C. M. (2016). Las redes sociales como herramientas de aprendizaje: algunas consideraciones. Revista de estudios latinos: RELat, (16), 225-256.

Zelada, Y. D. (2020). Plataformas Learning y TI en Programas de Postgrado, EVA: Una propuesta para el aprendizaje. Iberoamerican Business Journal, 3(2), 74-95.

\section{Anexos}

\section{CUESTIONARIO}

\begin{tabular}{|c|c|}
\hline \multicolumn{2}{|r|}{$\begin{array}{l}\text { Encuesta } \\
\text { Objetivo: identificar las herramientas tecnológicas usadas por la población Wayuu con el fin de } \\
\text { superar las barreras y dificultades presentadas para su manejo en el corregimiento de Nazareth } \\
\text { Municipio de Uribía Departamento de La Guajira, Colombia. }\end{array}$} \\
\hline \multicolumn{2}{|r|}{$\begin{array}{l}\text { Contestar todas las preguntas: } \\
\text { Se debe marcar, una sola opción con una X en la escala numérica sin repetir ninguna, de acuerdo al } \\
\text { orden de importancia que usted considere: Totalmente de Acuerdo (5). De Acuerdo (4). Ni de } \\
\text { Acuerdo ni en desacuerdo (3), En Desacuerdo (2), Totalmente en Desacuerdo(1) }\end{array}$} \\
\hline & \\
\hline ? & $\begin{array}{l}\text { sabe comparar con otros usuari } \\
\text { I }\end{array}$ \\
\hline 3 & 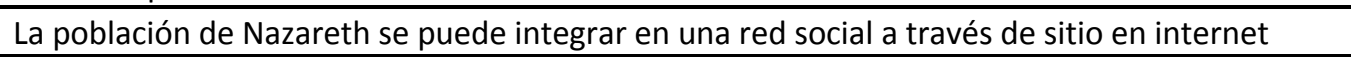 \\
\hline 4 & importa \\
\hline 5 & pok \\
\hline 6 & ción de crear, compartir, faciliț \\
\hline 7 & $\begin{array}{l}\text { El uso de las TIC ha impulsado una serie de cambio en el proceso de aprendizaje de la población } \\
\text { Wayuu }\end{array}$ \\
\hline & El proceso de enseñanza y aprendizaje han sido significativo en la población Wayuu \\
\hline & 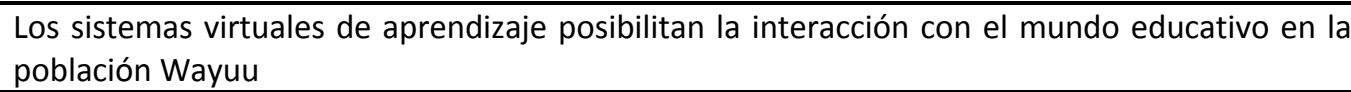 \\
\hline
\end{tabular}

Esta obra está bajo una Licencia Creative Commons Attribución-NoCommercial 4.0 International

(cc) EY-NC 